\title{
MULTI-PHYSICS COUPLING ANALYSIS OF ROPE-SEALED STRUCTURES WITH BRAIDED CERAMIC FIBRES BY ELEMENT DIFFERENTIAL METHOD
}

\author{
YONG-TONG ZHENG ${ }^{1}$, XIAO-WEI GAO ${ }^{1}$, SHENG LIU ${ }^{2}$, YAN-JING MAN ${ }^{2}$ \& KAI YANG ${ }^{1}$ \\ ${ }^{1}$ State Key Laboratory of Structural Analysis for Industrial Equipment, Dalian University of Technology, China. \\ ${ }^{2}$ Science and Technology on Scramjet Laboratory, Beijing Power Machinery Institute, China.
}

\begin{abstract}
With the rapid development of hypersonic vehicles in recent years, high-temperature seal technology has become more and more essential. Recently, a rope-sealed structure with braided ceramic fibres has been designed for hypersonic vehicles. The ceramic fibres in the structure have the characteristics of high temperature strength, so that they make the sealed structure suitable for working under a high temperature. Meanwhile, when subjected to an external force, braided fibres can produce a buffer force at the ceramic interface, so that it can maintain the good performance of the whole sealed structure. But up to now, only a few researches have been conducted on this kind of structures. In this paper, a simplified thermal-mechanical seepage coupling model is proposed to simulate the complicated physical process for this kind of structures. Meanwhile, a new numerical method called element differential method (EDM) is used to calculate the coupling problem because it has great advantages in solving multiphysics coupling problems. What is more, some experiments are used to obtain the leakages when the sealed structure is under service. And finally, by referring the experimental results, the authors establish a series of material parameter relationships for the sealed structure and also verify the reasonability of the proposed multi-physics coupling model.
\end{abstract}

Keywords: braided ceramic fibre rope, element differential method, hypersonic vehicle, sealed structure, seepage problem.

\section{INTRODUCTION}

High-temperature seal technology is one of the most important technologies in modern industry. Sealing technology is of great significance to the safety of industrial equipment and for energy saving and environmental protection. In recent years, with the rapid development of hypersonic aircrafts, high-temperature seal technology has become essential for preventing high-temperature air from entering the aircrafts and damaging the key components of the equipment.

In the hypersonic vehicles, the most commonly used seals are the ceramic sheets. Recently, a flexible seal strip with many braided ceramic fibres [1] was designed to fit in the new kind of dynamic sealed structures, as shown in Fig. 1. The ceramic fibres in the strip have the characteristics of high-temperature strength, by which they make the strip suitable for working under high temperature. Meanwhile, when subjected to an external force, the braided fibres can produce a buffer force at the ceramic interface, so that it can maintain the good performance of the whole sealed structure. This kind of ceramic fibre seal strips works well under high temperature, high pressure and frequent friction conditions, but it can only adapt to the gaps with a small change because its deformation only depends on the elasticity of braided fibres and the porosity of itself.

For the numerical analysis of the dynamic sealed structures, the finite element method (FEM) is the most commonly used method. Fern et al. [2] used ANSYS to simulate the mechanical performance of the valve stem seals, providing the basis of the valve stem seal design. Li et al. [3] introduced rod elements and established an axial symmetry model to 


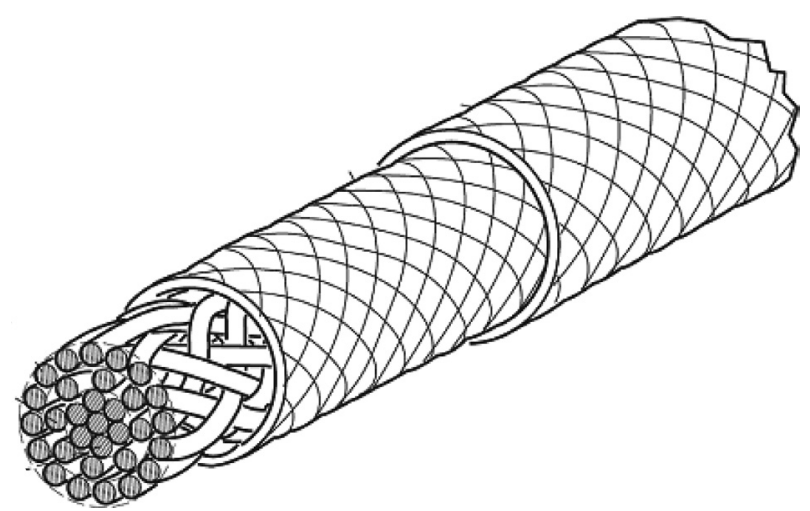

Figure 1: The seal strip with many braided ceramic fibres.

compute mechanical seal face deformation. Nikas et al. [4-8] developed a numerical model to study the sealing performance of rectangular elastomeric seals for reciprocating piston rods used in linear hydraulic actuators. Chen et al. $[9,10]$ used a large FEM program MARC/ Mentat320 to analyse the mechanical performance of the O-ring seals when they are installed and working with fluid. They also studied the mechanical performance of the step-ring seals [10]. The studies mentioned above show that a few researches have been conducted on the braided ceramic fibre seal strips. Actually, analysing the seal strips accurately is much more difficult than analysing most of other seals because the interactions between the fibres and fluids or between two fibres, and moreover, the anisotropy of fibres should be taken into consideration. So it is necessary to establish a simplified model for this type of structures and use a simple numerical method.

In recent years, Gao et al. [11-20] proposed a new strong-form numerical method called element differential method (EDM). Like traditional FEM, EDM also uses isoparametric elements to discretize the computational domain and approximate physical variables. In EDM, the element internal nodes use the directly discretized governing equations, while the boundary nodes use the discretized flux (or surface traction or velocity) equilibrium equations. Compared with FEM, the EDM does not need integrations, and it is easy to set up the discretized equations for complicated multi-physics coupling problems.

In this paper, a simplified thermal-mechanical seepage coupling model is proposed to simulate the complicated physical coupling process for braided ceramic fibre seal strips. Meanwhile, the new numerical method EDM is used to discretize the equations. Besides, some related experiments are also used to measure the leakage of the seal strip. By the inverse analysis from the experimental results and some assumptions, finally, the material parameter relationships are obtained, which cannot be easily measured from the experiments and can be used for further research about the seal strips. Meanwhile, these processes will also verify the reasonability of the mathematical model and show the ability of EDM in analysing multi-physics coupling problems.

\section{GOVERNING EQUATIONS OF ROPE SEAL STRIP WITH BRAIDED CERAMIC FIBRES}

As mentioned in Section 1, it is quite complicated to consider the interactions between fibres and fluid or between two fibres in detail. So, a simplified way is adapted in this paper in which the complicated physical processes are regarded as the coupling of elasticity, heat conduction 
problem and seepage problem. Without considering the body force, the elasticity equations can be written as:

$$
\begin{gathered}
\frac{\partial \sigma_{i j}}{\partial x_{j}}=0 \\
\sigma_{i j}=C_{i j k l} \varepsilon_{k l}-k \delta_{i j}\left(T-T_{0}\right)-b \delta_{i j}\left(P-P_{0}\right), \\
\varepsilon_{i j}=\frac{1}{2}\left(\frac{\partial u_{i}}{\partial x_{j}}+\frac{\partial u_{j}}{\partial x_{i}}\right),
\end{gathered}
$$

in which $\sigma$ represents the stress tensor, $x_{j}$ is the coordinate of $j$-th direction, $C$ is the elastic constitutive tensor, $\varepsilon$ is the strain tensor, $u_{i}$ is the displacement of $i$-th direction, $T$ is temperature, $P$ is seepage pressure, $T_{0}$ and $P_{0}$ are the temperature and seepage pressure when the material has no deformation, respectively, $\delta_{i j}$ is Kronecher delta, $b$ is Biot coefficient, $k$ is the coefficient of thermal stress and the repeated subscripts $i, j, k$ and $l$ represent summation. The two kinds of boundary conditions of elasticity are

$$
\begin{gathered}
u_{i}=\bar{u}_{i}, \\
\sigma_{i j} \cdot n_{j}=\bar{t}_{i},
\end{gathered}
$$

where $\bar{u}_{i}$ and $\bar{t}_{i}$ are the prescribed displacement and surface traction, respectively.

If only the steady state is considered, the heat conduction equation can be written as:

$$
\frac{\partial}{\partial x_{i}}\left(K \cdot \frac{\partial T}{\partial x_{i}}\right)=0,
$$

where $K$ is thermal conductivity. In this paper, there are two kinds of boundary conditions of heat conduction involved as follows:

$$
\begin{gathered}
T=\bar{T} \\
-K \cdot \frac{\partial T}{\partial x_{i}} \cdot n_{i}=\bar{q},
\end{gathered}
$$

where $\bar{T}$ and $\bar{q}$ are the prescribed temperature and heat flux, respectively.

Usually, for the problem discussed in this paper, the characteristic time of seepage equation is much less than that of heat conduction equation; so, there is no need to consider the time term of seepage equation, which can be expressed as:

$$
\frac{\partial}{\partial x_{i}}\left[\frac{a}{v} \frac{\partial P}{\partial x_{i}}\right]=0,
$$

where $\alpha$ is permeability and $v$ is the dynamic viscosity coefficient of fluid. Also, there are two kinds of boundary conditions of seepage as follows:

$$
P=\bar{P}
$$




$$
-\frac{a}{v} \cdot \frac{\partial P}{\partial x_{i}} \cdot n_{i}=\bar{v},
$$

where $\bar{P}$ and $\bar{v}$ are the prescribed seepage pressure and velocity, respectively.

Except the parameters in three governing equations, one important parameter called porosity should be defined, which affects the parameters of the governing equations and is also affected by volumetric strain, i.e.

$$
\Phi=f_{1}\left(\varepsilon_{v}\right)
$$

where $\Phi$ represents porosity and $\varepsilon_{v}$ is the volumetric strain which can be calculated as follows:

$$
\varepsilon_{v}=\varepsilon_{11}+\varepsilon_{22}+\varepsilon_{33} \text {. }
$$

In eqn (2), elastic constitutive tensor $C$ is a fourth-order tensor and can be expressed by the formula

$$
C_{i j k l}=\lambda \delta_{i j} \delta_{k l}+\mu\left(\delta_{i k} \delta_{j l}+\delta_{i l} \delta_{j k}\right),
$$

in which $\lambda$ and $\mu$ are the Lamé constants and they can be expressed by the formula

$$
\begin{gathered}
\lambda=\frac{E v}{(1+v)(1-2 v)}, \\
\mu=\frac{E}{2(1+\nu)},
\end{gathered}
$$

where $E$ and $v$ are the elastic modulus and Poisson ratio, respectively. Coefficient of thermal stress can be calculated by the formula

$$
k=(2 \lambda+3 \mu) \beta
$$

in which $\beta$ is the coefficient of thermal expansion. Obviously, it is quite hard to get the elastic modulus of the braided ceramic fibre rope. In this paper, the elastic modulus is assumed to be a function of porosity and temperature, which can be calculated using the formula

$$
E=E_{0} f_{2}(\Phi) f_{3}(T) \text {. }
$$

Similarly, permeability and thermal conductivity can also be expressed as the functions of porosity:

$$
\begin{gathered}
a=f_{4}(\Phi), \\
K=(1-\Phi) K_{s}+\Phi K_{l},
\end{gathered}
$$

in which $K_{s}$ and $K_{l}$ are the thermal conductivities of solid and fluid, respectively.

It can be found that the three governing equations affect each other; therefore, the physical process in the braided ceramic fibre rope is a nonlinear multi-physics coupling problem.

\section{USING EDM TO DISCRETIZE THE GOVERNING EQUATIONS}

EDM is a collocation method. It uses isoparametric elements to discretize the governing equations, just like the traditional FEM does. In this paper, nine-node Lagrange element is used, which is shown in Fig. 2. Generally, in Lagrange element, physical variable $u$ and 


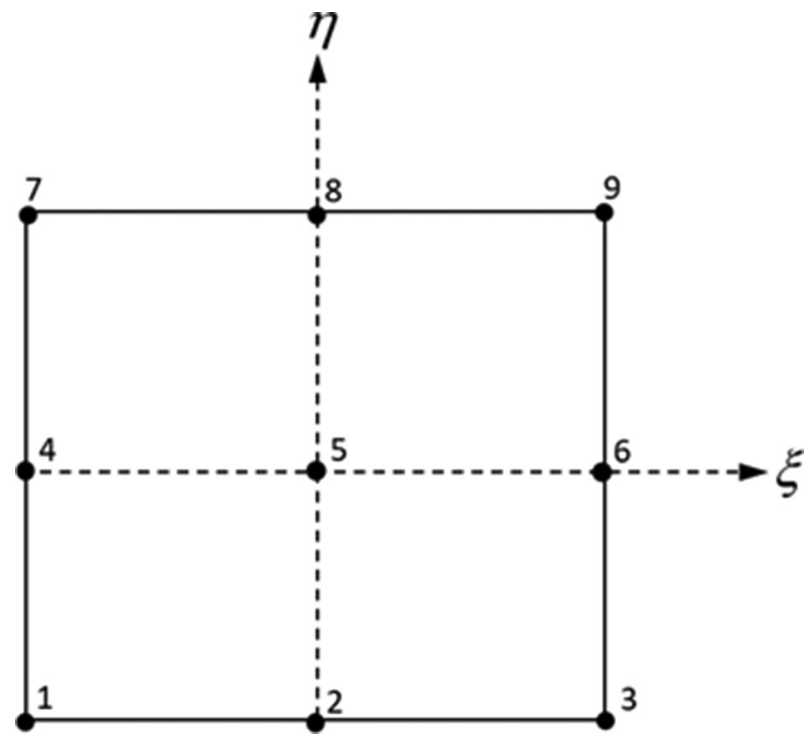

Figure 2: Nine-node Lagrange element.

coordinate $x_{i}$ of any point can be represented by linear combinations of physical variables and coordinates on nine nodes, respectively, i.e.

$$
\begin{gathered}
u=N_{a} u^{a}, \\
x_{i}=N_{a} x_{i}^{a},
\end{gathered}
$$

where $N_{\alpha}$ is called the shape function of node $\alpha$. Similarly, the first- and second-order derivate of physical variable can also be represented by the formula

$$
\begin{gathered}
\frac{\partial T}{\partial x_{i}}=\frac{\partial N_{a}}{\partial x_{i}} T^{a}, \\
\frac{\partial^{2} T}{\partial x_{i} \partial x_{j}}=\frac{\partial^{2} N_{a}}{\partial x_{i} \partial x_{j}} T^{a} .
\end{gathered}
$$

The formula of shape functions and its first or second order can be obtained in the reference papers [11,12].

For the element internal nodes, such as node 5 in Fig. 2, their equations are the discretized governing equations, i.e.

$$
\begin{aligned}
& {\left[\frac{\partial N_{\beta}}{\partial x_{j}} C_{i j k l}^{\beta} \frac{\partial N_{a}}{\partial x_{l}}+C_{i j k l} \frac{\partial^{2} N_{a}}{\partial x_{l} \partial x_{j}}\right] u_{k}^{a}+\frac{\partial N_{a}}{\partial x_{i}}\left(k\left(T-T_{0}\right)\right)^{a}+\frac{\partial N_{a}}{\partial x_{i}}\left(b\left(P-P_{0}\right)\right)^{a}=0, } \\
& {\left[\frac{\partial N_{\beta}}{\partial x_{i}} K^{\beta} \frac{\partial N_{a}}{\partial x_{i}}+K \frac{\partial^{2} N_{a}}{\partial x_{i}^{2}}\right] T^{a}=0, }
\end{aligned}
$$




$$
\left[\frac{\partial N_{\beta}}{\partial x_{i}} \frac{a^{\beta}}{v} \frac{\partial N_{a}}{\partial x_{i}}+\frac{a}{v} \frac{\partial^{2} N_{a}}{\partial x_{i}^{2}}\right] P^{a}=0 .
$$

Except the element internal nodes, the other nodes in the Lagrange element are the element boundary nodes. For element boundary nodes, the surface traction or heat flux or velocity equilibrium equations should be satisfied, i.e.

$$
\begin{gathered}
\sum_{e=1}^{N}\left[\left(C_{i j k l} \frac{\partial N_{a}}{\partial x_{l}} u_{k}^{a}-k\left(T-T_{0}\right) \delta_{i j}-b\left(P-P_{0}\right) \delta_{i j}\right)_{f=1}^{M} n_{j}^{f}\right]=\sum_{e=1}^{N} \sum_{f=1}^{M} \overline{t_{i}^{f}} \text { or } 0, \\
-\sum_{e=1}^{N}\left[\left(K \frac{\partial N_{a}}{\partial x_{i}} T^{a}\right) \sum_{f=1}^{M} n_{i}^{f}\right]=\sum_{e=1}^{N} \sum_{f=1}^{M} \overline{q^{f}} \text { or } 0, \\
-\sum_{e=1}^{N}\left[\left(\frac{a}{v} \frac{\partial N_{a}}{\partial x_{i}} P^{a}\right) \sum_{f=1}^{M} n_{i}^{f}\right]=\sum_{e=1}^{N} \sum_{f=1}^{M} \overline{v^{f}} \text { or } 0,
\end{gathered}
$$

in which $N$ is the number of elements that this node is involved in, $M$ is the number of surfaces in element $e$ that this node is located on and $e$ and $f$ are the element number and surface number, respectively. To illustrate the equilibrium equations in detail, three kinds of common cases of element boundary nodes are shown in Fig. 3.

Based on eqns (25) (30), for 2D problem, one node has two elasticity, one heat conduction and one seepage equation (four equations in total). A nonlinear system can be obtained by listing these equations together, i.e.
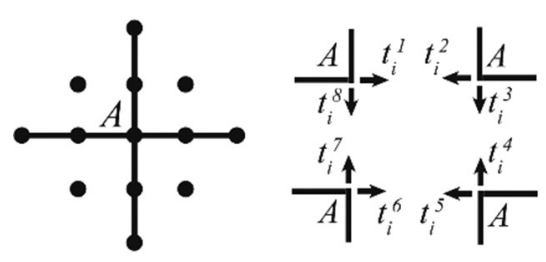

$$
\begin{aligned}
& t_{i}^{1}+t_{i}^{2}=0 \\
& t_{i}^{3}+t_{i}^{4}=0 \\
& t_{i}^{5}+t_{i}^{6}=0 \\
& t_{i}^{7}+t_{i}^{8}=0
\end{aligned} \quad \begin{gathered}
t_{i}^{1}+t_{i}^{2}+\ldots+t_{i}^{8}=0 \\
i=1,2
\end{gathered}
$$

(a) case 1
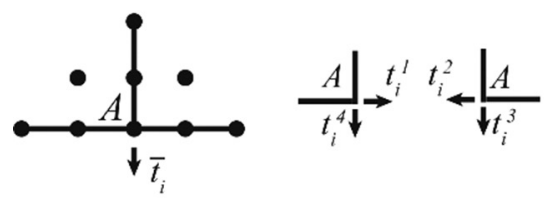

$$
\begin{aligned}
& t_{i}^{1}+t_{i}^{2}=0 \\
& t_{i}^{3}=\bar{t}_{i} \\
& t_{i}^{4}=\bar{t}_{i}
\end{aligned} t_{i}^{1+}+t_{i}^{2}+t_{i}^{3}+t_{i}^{4}=2 \bar{t}_{i}
$$

(b) case 2
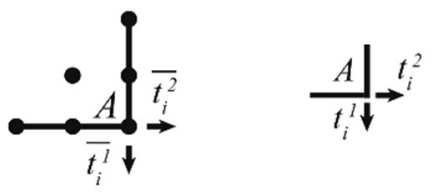

$$
\begin{gathered}
t_{i}^{l}=\overline{t_{i}^{l}} \\
t_{i}^{2}=\overline{t_{i}^{2}}
\end{gathered} \begin{gathered}
t_{i}^{l}+t_{i}^{2}=\overline{t_{i}^{1}}+\overline{t_{i}^{2}} \\
i=1,2
\end{gathered}
$$

(c) case 3

Figure 3: Three kinds of common cases of element boundary nodes. 


$$
[A(x)]\{x\}=\{b\}
$$

in which $[A]$ is the coefficient matrix, $\{x\}$ is the solution vector and $\{b\}$ is the right-side vector. Usually, Newton-Raphson method is used to solve it. After solving it, we can obtain displacements, temperatures and seepage pressures of all nodes.

\section{LEAKAGE PERFORMANCE EXPERIMENT ABOUT THE SEAL STRIP WITH BRAIDED CERAMIC FIBRES}

The leakage performance experiments are the important references for numerical computation. As shown in Fig. 4, the experiments are aimed to obtain the leakages when the seal strip is compressed by $20 \%$ under the conditions of four different pressures and three different temperatures. The experimental processes are to compress the seal strip from the right side and blow air from the top and measure the leakage from the bottom. In Fig. 5 is presented a

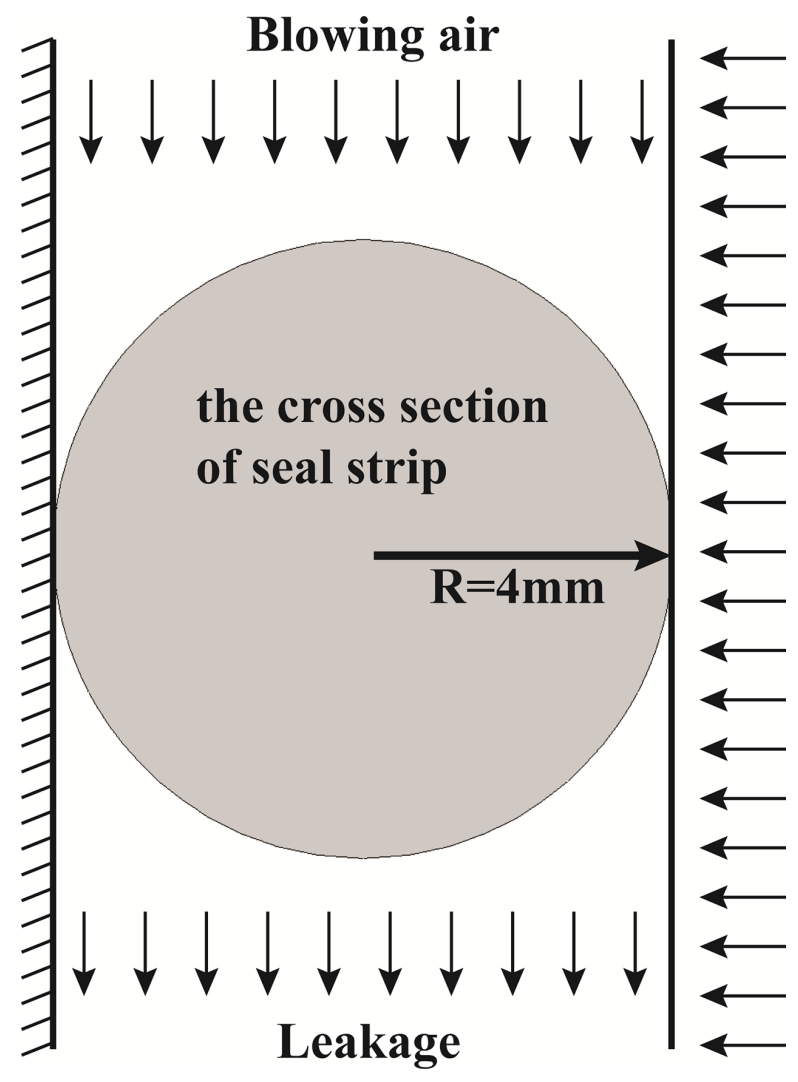

Figure 4: Schematic diagram of the experiment.

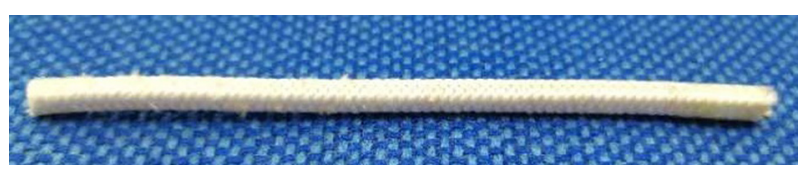

Figure 5: Braided ceramic fibre seal strip. 


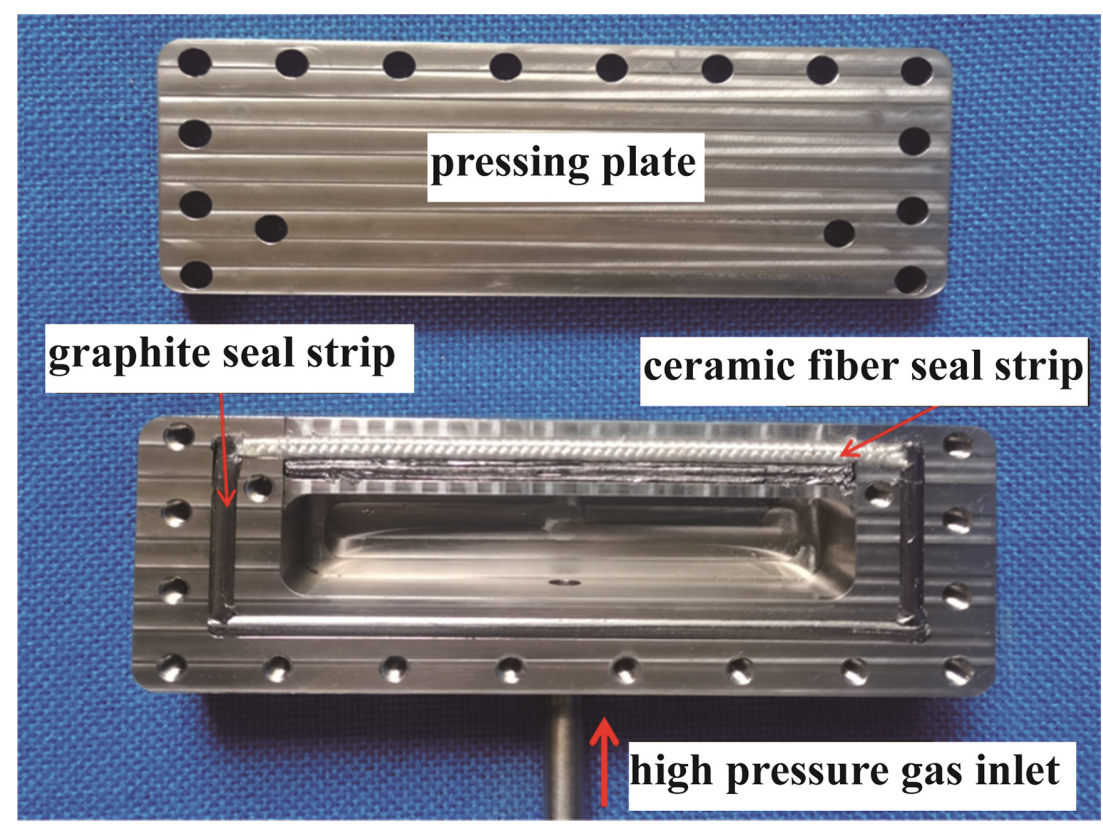

Figure 6: Experimental facility.

Table 1: Leakage results of the experiment $(\mathrm{g} /[\mathrm{m} \cdot \mathrm{s}])$.

\begin{tabular}{lllll}
\hline $\begin{array}{l}\text { pressure difference } \\
\text { (MPa) }\end{array}$ & $\mathbf{3 0 0 ~ K}$ & $\mathbf{6 0 0} \mathbf{~ K}$ & $\mathbf{9 0 0} \mathbf{~ K}$ & $\mathbf{1 0 7 0} \mathbf{~ K}$ \\
\hline 0.1 & 1.542 & 1.76 & 1.612 & 1.616 \\
0.2 & 3.334 & 3.434 & 3.296 & 3.27 \\
0.3 & 5.538 & 5.42 & 5.082 & 5.058 \\
\hline
\end{tabular}

picture of the braided ceramic fibre seal strip. Figure 6 shows the experimental facilities. The leakage results of the experiments are the average of each cross section and each second, as shown in Table 1.

\section{NUMERICAL SIMULATION ABOUT THE SEAL STRIP WITH BRAIDED CERAMIC FIBRES}

To compute the seal strip, first, the computational model needs to be set up. For convenience, the authors only consider the cross section far away from two sides of seal strip, which can be regarded as the plane strain state. Referring to Fig. 4, the left and right contact parts of the cross section can be calculated by the compression rate. Then, four points are obtained to separate the circle into four parts. On taking the centre of the cross section as the origin and setting up the Cartesian coordinate system as shown in Fig. 7, the coordinates of four points are $(-3.6,1.744) \mathrm{mm},(-3.6,-1.744) \mathrm{mm},(3.6,1.744) \mathrm{mm}$ and $(3.6,-1.744) \mathrm{mm}$, respectively. The displacements of the left part satisfy the equation $u=4-x$, while those of the right part satisfy the equation $u=0.2+4-x$. The seepage pressure of the upper part is $0.2,0.3$ or $0.4 \mathrm{MPa}$, while that of the lower part is $0.1 \mathrm{MPa}$. The temperatures of the whole model are the same, which can be $300,600,900$ or $1070 \mathrm{~K}$. 


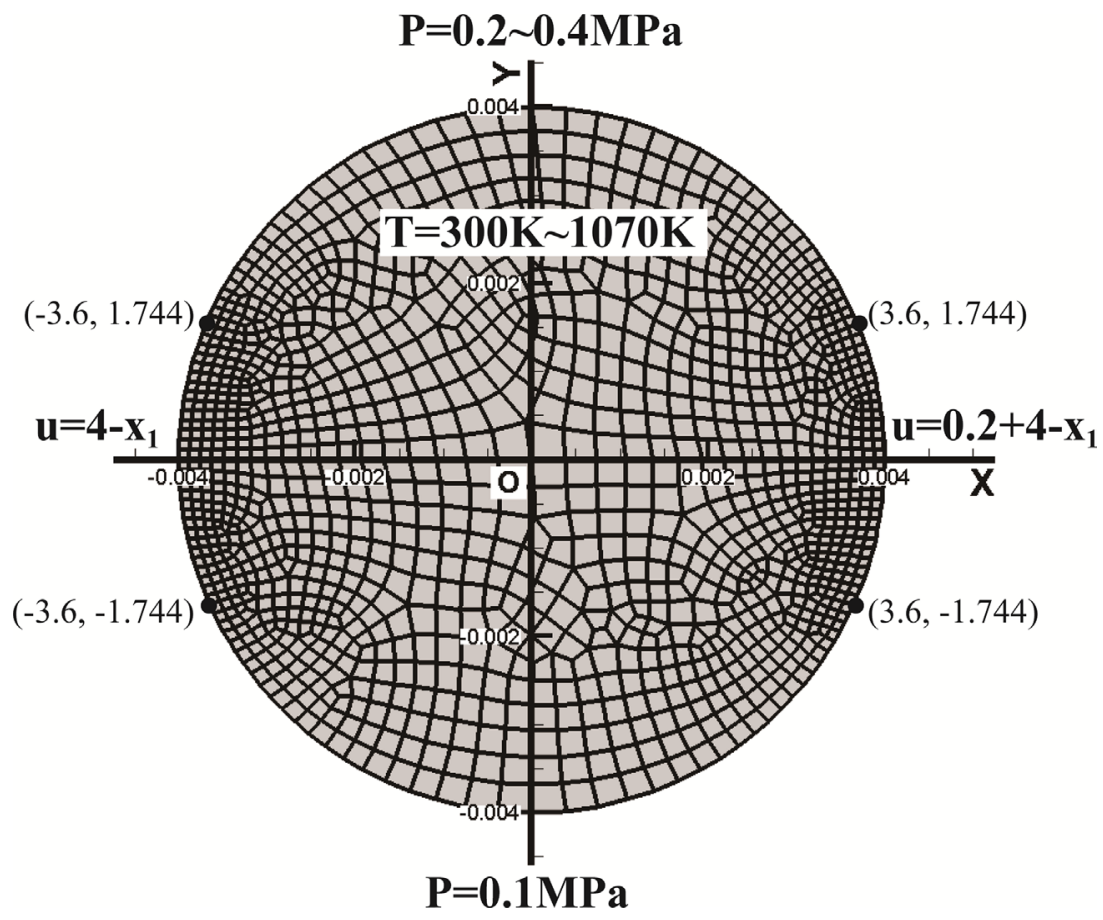

Figure 7: Computational model and mesh.

The leakage $Q$ can be calculated by the formula

$$
Q=\int_{l} \vec{v} \cdot \vec{n} d l,
$$

where $l$ is the arc from point $(-3.6,-1.744) \mathrm{mm}$ to point $(3.6,-1.744) \mathrm{mm}, \vec{n}$ is the outer normal vector of $l$ and $\vec{v}$ is the velocity vector on $l$.

Generally, it is hard to get some of the material parameters and their relationships by the experiments. In order to settle them down, the authors combined the experiences, assumptions and the inverse analysis from the experimental results. They are shown below.

1. Elasticity modulus

Referring to eqn (18), $E_{0}$ is set to $3.5 \mathrm{GPa}$ and

$$
\begin{gathered}
f_{2}(\Phi)=\frac{e^{-5 \Phi}-e^{-5}}{1-e^{-5}}, \\
f_{3}(T)=1-0.0005\left(T-T_{0}\right) .
\end{gathered}
$$

2. Poisson ratio is set to 0.22 .

3. Heat conductivity

Referring to eqn (20), $K_{s}=25 \mathrm{~W} /(\mathrm{m} \cdot \mathrm{K})$ and $K_{l}=0.02 \mathrm{~W} /(\mathrm{m} \cdot \mathrm{K})$.

4. Dynamic viscosity coefficient of the fluid is $1.79 \times 10^{-5} \mathrm{~Pa} \cdot \mathrm{s}$.

5. Permeability

Referring to eqn (19), 
Table 2: Leakage results of computation $(\mathrm{g} /[\mathrm{m} \cdot \mathrm{s}])$.

\begin{tabular}{lllll}
\hline $\begin{array}{l}\text { pressure difference } \\
\text { (MPa) }\end{array}$ & $\mathbf{3 0 0} \mathbf{~ K}$ & $\mathbf{6 0 0} \mathbf{~ K}$ & $\mathbf{9 0 0} \mathbf{~ K}$ & $\mathbf{1 0 7 0} \mathbf{~ K}$ \\
\hline 0.1 & 1.748712728 & 1.725174102 & 1.702227437 & 1.689530789 \\
0.2 & 3.401038490 & 3.376207859 & 3.352376061 & 3.339512006 \\
0.3 & 5.054916439 & 5.029128657 & 5.004930785 & 4.992343699 \\
\hline
\end{tabular}

Table 3: Error of computational results and experiment results.

\begin{tabular}{lllll}
\hline $\begin{array}{l}\text { pressure difference } \\
\text { (MPa) }\end{array}$ & $\mathbf{3 0 0} \mathbf{K}$ & $\mathbf{6 0 0 ~ K}$ & $\mathbf{9 0 0} \mathbf{K}$ & $\mathbf{1 0 7 0} \mathbf{~ K}$ \\
\hline 0.1 & $13.41 \%$ & $1.98 \%$ & $5.60 \%$ & $4.55 \%$ \\
0.2 & $2.01 \%$ & $1.68 \%$ & $1.71 \%$ & $2.13 \%$ \\
0.3 & $8.72 \%$ & $7.21 \%$ & $1.52 \%$ & $1.30 \%$ \\
\hline
\end{tabular}

$$
f_{4}(\Phi)=(5+45 \Phi) \times 10^{-14} \mathrm{~m}^{2}
$$

6. Porosity

Referring to eqn (12),

$$
f_{1}\left(\varepsilon_{v}\right)=\frac{1}{1.42857+e^{\left(-10 \varepsilon_{v}-1.30155\right)}} .
$$

7. Thermal expansion is set to $7.5 \times 10^{-6} \mathrm{~K}^{-1}$.

8. Biot coefficient is 1.0 .

Using the model and materials mentioned above, the computational results can be obtained, which are shown in Table 2.

To compare the experimental results with the computational results, an error table was used (see Table 3). It can be found that the maximum error $13.41 \%$ occurs at the temperature $300 \mathrm{~K}$ and a pressure difference $0.1 \mathrm{MPa}$, although most of the errors are around $2 \%$. If the possible measurement errors and computational errors are taken into consideration, the mathematical model and the assumed material parameter relationships can be used to predict the leakage of the braided ceramic fibre seal strip when it works especially under a high temperature and high pressure difference.

\section{CONCLUSION}

In this paper, a new mathematical model is proposed to simulate the rope seal strips with braided ceramic fibres. Meanwhile, EDM is used to discretize the governing equations to set up the algebraic equations. Besides, the related experiments are also used to measure the leakage of the seal strip. By the inverse analysis from the experimental results and some assumptions, finally, the material parameters are obtained, which cannot be easily measured from the experiments and can be used for the further researches. Of course, the agreement reached between the experiments and the computations also verifies the reasonability of the 
proposed mathematical model and shows the ability of EDM in analysing multi-physics coupling problems.

\section{CONFLICT OF INTEREST}

The authors declare that they have no conflict of interests.

\section{ACKNOWLEDGEMENT}

The National Natural Science Foundation of China (Grant Nos 11672061, 12072064, 12072060 ) is gratefully acknowledged for the support given for this investigation.

\section{REFERENCE}

[1] Dunlap, P.H., Steinetz, B.M. \& Curry, D.M., Rudder/fin seal investigations for the X-38 re-entry vehicle. 36th Joint Propulsion Conference and Exhibit cosponsored by AIAA/ ASME/SAE/ASEE, 2000.

[2] Fern, A.G., Mason-Jones, A., Pham, D.T. \& Wang, J., Finite element analysis of a valve stem seal. International Congress \& Exposition, 1998.

[3] Kun, L., Liming, Y., Zhaoshan, W. \& Chunyang, W., Study on face deformation of mechanical seal. Lubrication Engineering, 2001.

[4] Nikas, G.K., Elastohydrodynamics and mechanics of rectangular elastomeric seals for reciprocating piston rods. Journal of Tribology, 125(1), pp. 60-69, 2003. https://doi. org/10.1115/1.1506316

[5] Nikas, G.K., Transient elastohydrodynamic lubrication of rectangular elastomeric seals for linear hydraulic actuators. ARCHIVE Proceedings of the Institution of Mechanical Engineers Part J Journal of Engineering Tribology 1994-1996 (vols 208-210), 217(6), pp. 461-473, 2003. https://doi.org/10.1243/135065003322620282

[6] Nikas, G.K. \& Sayles, R.S., Nonlinear elasticity of rectangular elastomeric seals and its effect on elastohydrodynamic numerical analysis. Tribology International, 37(3), pp. 651-660, 2004. https://doi.org/10.1016/j.triboint.2004.02.002

[7] Nikas, G.K. \& Sayles, R.S., Study of leakage and friction of flexible seals for steady motion via a numerical approximation method. Tribology International, 39(9), pp. 921-936, 2006. https://doi.org/10.1016/j.triboint.2005.09.003

[8] Nikas, G.K., Eighty years of research on hydraulic reciprocating seals: Review of tribological studies and related topics since the 1930s. Proceedings of the Institution of Mechanical Engineers, Part J: Journal of Engineering Tribology, 224(1), pp. 1-23, 2010. https://doi.org/10.1243/13506501jet607

[9] Guo-ding, C., Haiser, H., Haas, W. \& Lechner, G., Analysis of elastomeric o-ring seals using the Finite Element Method. Mechanical Science and Technology, 19(5), pp. 740 $741,2000$.

[10] Guo-ding, C., Haiser, H., Haas, W. \& Lechner, G., Study of mechanical performances of step seals. Mechanical Science and Technology, 19(6), pp. 920-922, 2000.

[11] Gao, X.-W., Li, Z.-Y., Yang, K., Lv, J., Peng, H.-F., Cui, M., Ruan, B. \& Zhu, Q.-H., Element differential method and its application in thermal-mechanical problems. International Journal for Numerical Methods in Engineering, 113(1), pp. 82-108, 2018. https://doi.org/10.1002/nme.5604

[12] Gao, X.-W., Huang, S.-Z., Cui, M., Ruan, B., Zhu, Q.-H., Yang, K., Lv, J. \& Peng, H.-F., Element differential method for solving general heat conduction problems. Inter- 
national Journal of Heat and Mass Transfer, 115(B), pp. 882-894, 2017. https://doi. org/10.1016/j.ijheatmasstransfer.2017.08.039

[13] Gao, X.-W., Liu, H.-Y., Xu, B.-B., Cui, M. \& Lv, J., Element differential method with the simplest quadrilateral and hexahedron quadratic elements for solving heat conduction problems. Numerical Heat Transfer Part B-Fundamentals, 73(4), pp. 206-224, 2018. https://doi.org/10.1080/10407790.2018.1461491

[14] Cui, M., Xu, B.-B., Lv, J., Gao, X.-W. \& Zhang, Y.W., Numerical solution of multidimensional transient nonlinear heat conduction problems with heat sources by an extended element differential method. International Journal of Heat and Mass Transfer, 126(A), pp. 1111-1119, 2018. https://doi.org/10.1016/j.ijheatmasstransfer.2018.05.100

[15] Yang, K., Jiang, G.-H., Li, H.-Y., Zhang, Z.-B. \& Gao, X.-W., Element differential method for solving transient heat conduction problems. International Journal of Heat and Mass Transfer, 127, pp.1189-1197, 2018. https://doi.org/10.1016/j.ijheatmasstransfer.2018.07.155

[16] Gao, X.-W., Liu, H.-Y., Lv, J. \& Cui, M., A novel element differential method for solid mechanical problems using isoparametric triangular and tetrahedral elements. Computers \& Mathematics with Applications, 78(11), pp. 3563-3585, 2019. https://doi. org/10.1016/j.camwa.2019.05.026

[17] Lv, J., Son, C. \& Gao, X.-W., Element differential method for free and forced vibration analysis for solids. International Journal of Mechanical Sciences, 151, pp. 828-841, 2019. https://doi.org/10.1016/j.ijmecsci.2018.12.032

[18] Lv, J., Shao, M.-J., Cui, M. \& Gao, X.-W., An efficient collocation approach for piezoelectric problems based on the element differential method. Composite Structures, 230(111483), 2019. https://doi.org/10.1016/j.compstruct.2019.111483

[19] Gao, X.-W., Zheng, Y.-T. \& Fantuzzi, N., Local least-squares element differential method for solving heat conduction problems in composite structures. Numerical Heat Transfer, Part B: Fundamentals, 77(6), pp. 441-460, 2020. https://doi.org/10.1080/10 407790.2020.1746584

[20] Zheng, Y.-T., Gao, X.-W., Lv, J. \& Peng, H.-F., Weak-form element differential method for solving mechanics and heat conduction problems with abruptly changed boundary conditions. International Journal for Numerical Methods in Engineering, 121(16), pp. 3722-3741, 2020. https://doi.org/10.1002/nme.6379 